\title{
Evaluación de los determinantes de la desnutrición crónica en el Perú
}

\section{Determinant assessment of chronic malnutrition in Peru}

\author{
Ricardo N. Villamonte Blas ${ }^{1}$ \\ rvillamonteb@unmsm.edu.pe \\ https://orcid.org/0000-0002-0759-9074 \\ Melquiades Mario Huamán Huarcaya ${ }^{2}$ \\ 20140629@aloe.ulima.edu.pe \\ https://orcid.org/0000-0002-0718-1559
}

Recibido: 07/12/2020, Aceptado: 26/03/2021

\begin{abstract}
RESUMEN
La importancia de la presente investigación es reconocer e incentivar el análisis de variables sociales y económicas que inciden en la desnutrición crónica infantil en niños menores de cinco años para el Perú en el período 2007 - 2018. Se busco comprobar si el modelo econométrico panel data es estadísticamente significativo. Para logarlo, se construyo un modelo a partir de recomendaciones de organismos internacionales y de estudios realizados por varios investigadores. Se concluye que el modelo econométrico evaluado es significativo. La diarrea crónica tiene en el modelo mayor incidencia que la anemia en la reducción de la desnutrición, como también el servicio de electrificación tiene un mayor coeficiente de incidencia que los servicios de saneamiento y agua, finalmente el quintil inferior y las zonas rurales afectan a la variable desnutrición corroborando la literatura empleada.
\end{abstract}

Palabras clave: desnutrición, diarrea, anemia, electrificación, saneamiento, agua, quintil, rural.

\section{ABSTRACT}

The importance of this research is to recognize and encourage the analysis of social and economic variables that affect chronic child malnutrition in children under five years of age for Peru in the period 2007 - 2018. It was sought to verify if the econometric panel data model is statistically significant. To achieve this, a model was built based on recommendations from international organizations and studies carried out by various researchers. It is concluded that the econometric model evaluated is significant. Chronic diarrhea has a higher incidence in the model than anemia in reducing malnutrition, as well as the electrification service has a higher incidence coefficient than the sanitation and water services, finally the lower quintile and rural areas affect the population. malnutrition variable corroborating the literature used.

\footnotetext{
${ }^{1}$ Facultad de Economía de la Universidad Nacional Mayor de San Marcos, Perú.

${ }^{2}$ Facultad de Economía de la Universidad Nacional Mayor de San Marcos, Perú.
} 


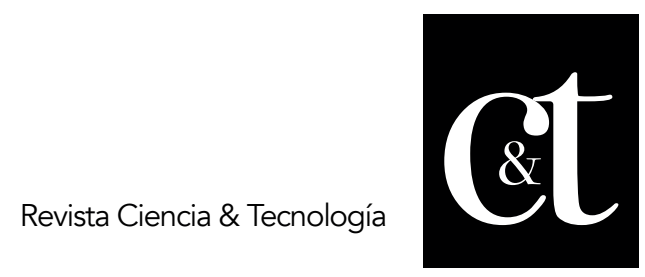

No. 30, 30 de abril de 2021

ISSN impreso: 1390 - 6321

ISSN online: 2661 - 6734

Keywords: malnutrition, diarrhea, anemia, electrification, sanitation, water, quintile, rural

\section{Introducción}

El informe de la UNICEF (1998) señala la importancia de la adecuada nutrición en la formación de la mente y el cuerpo de los niños para el futuro. Ellos de la mano de políticas sociales y económicas que resguarden su desarrollo fisiológico tienen la posibilidad de mejorar su futuro al consolidar sus capacidades productivas. El informe concluye señalando a la pobreza como la causa principal de la desnutrición infantil. Sin embargo, pese a ser una verdad común que la falta de recursos está estrechamente vinculada a los niveles de desnutrición, ésta solo representa una parte de una realidad más compleja.

El estudio de la Organización Panamericana de la Salud (2003) señala que un mal estado de salud se traduce en mala productividad para el que la padece, una vida laboral más corta y una menor remuneración por días perdidos por enfermedad

La investigación de la UNICEF (2008) señala la gravedad del problema de la desnutrición crónica para América Latina y el Caribe, la cual afecta el crecimiento y desarrollo de la niñez, lo cual se traduciría en menor calidad y cantidad de capital humano en los distintos países.

Según el informe de la Organización Mundial de la Salud (2014), la desnutrición se viene acentuando como uno de los problemas más relevantes a nivel mundial, siendo partícipe de cerca de un tercio de todas las muertes infantiles. Esta enfermedad está tomando fuerza en países pobres especialmente en las zonas donde los servicios de salud, educación y saneamiento son débiles.

En una pronta respuesta por parte de la OMS (2014), sugiere estrategias que incluyen establecer políticas apropiadas en materia de nutrición y alimentación, adoptar tendencias mundiales de nutrición para tomar decisiones, proporcionar asesoramiento nutricional para ejecutar las correctivas mediante suplementos dependiendo de cada circunstancia que se vaya a encontrar en cada país miembro para que de esa manera sea más amigable y fructífera la intervención.

Para la Organización Panamericana de la Salud (2018), el desarrollo y crecimiento adecuado de los niños se ha convertido es una pieza fundamental en el desarrollo integral de cualquier nación. La desnutrición tiene un impacto negativo significativo, lo que conduce a un estancamiento de la pobreza ya que repercute a futuro en pérdidas directas en la productividad, pérdidas económicas debido al bajo desarrollo de criterio y razonamiento, al bajo nivel de habilidades que se gesta desde cuando uno es niño.

La Organización de las Naciones Unidad para la Alimentación y la Agricultura (FAO) (2019) reconoce que el mundo debe enfocar esfuerzos conjuntos para enfrentar a un enemigo que cada año afecta a más personas, teniendo como eje principal el hambre, la malnutrición y la inseguridad alimentaria.

En el Gráfico No 1 se observa el diagrama base que utiliza UNICEF (2008) que deja a un lado la visión tradicional que el problema de la desnutrición infantil se limite sólo al sector salud, sino que evidencia la influencia de múltiples sectores como el económico, social y político en el crecimiento y desarrollo de los niños.

\section{6}

Villamonte, Huaman. Evaluación de los determinantes de la desnutrición crónica en el Perú 


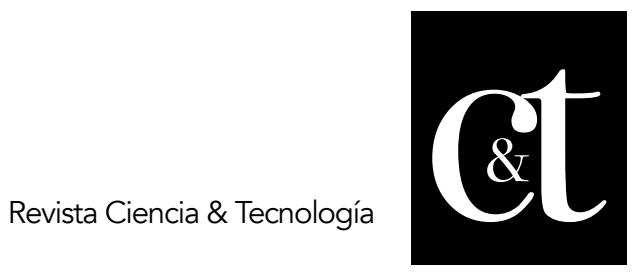

No. 30, 30 de abril de 2021

ISSN impreso: 1390 - 6321

ISSN online: 2661 - 6734

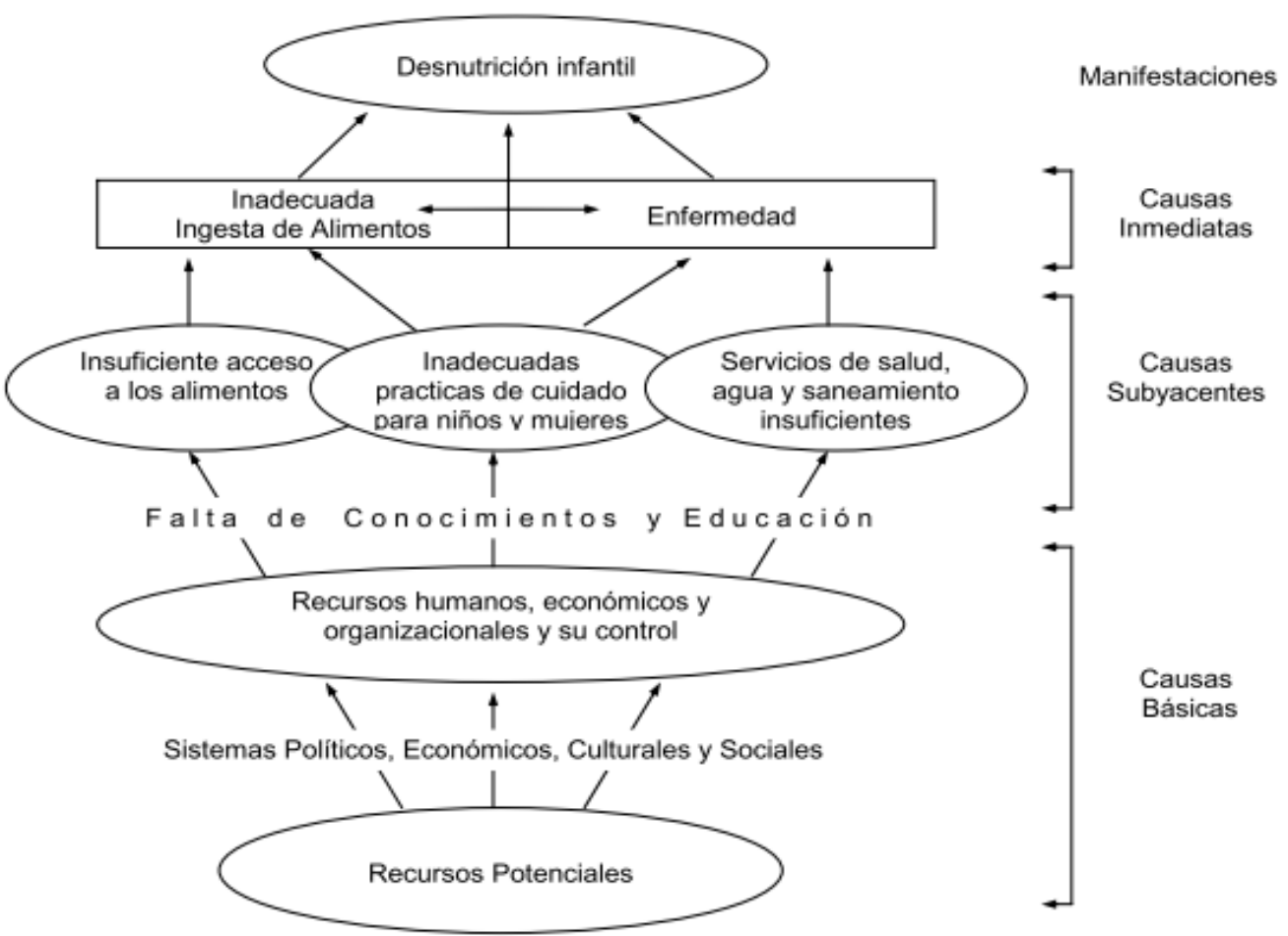

Gráfico 1. Modelo de causalidad de la Desnutrición Crónica Infantil

Fuente: UNICEF, TACRO Panamá, 2008.

En el caso peruano el Ministerio de Salud implemento el Plan Nacional de Salud que consideró los aportes de los organismos internacionales y nacionales, en el Gráfico 2 se aprecia el modelo causal de la anemia en el Perú el cual se fue implementando y ejecutando mediante dispositivos legales del sector y de los sectores vinculados, siempre se lucho por la articulación de todos las instituciones involucradas. 


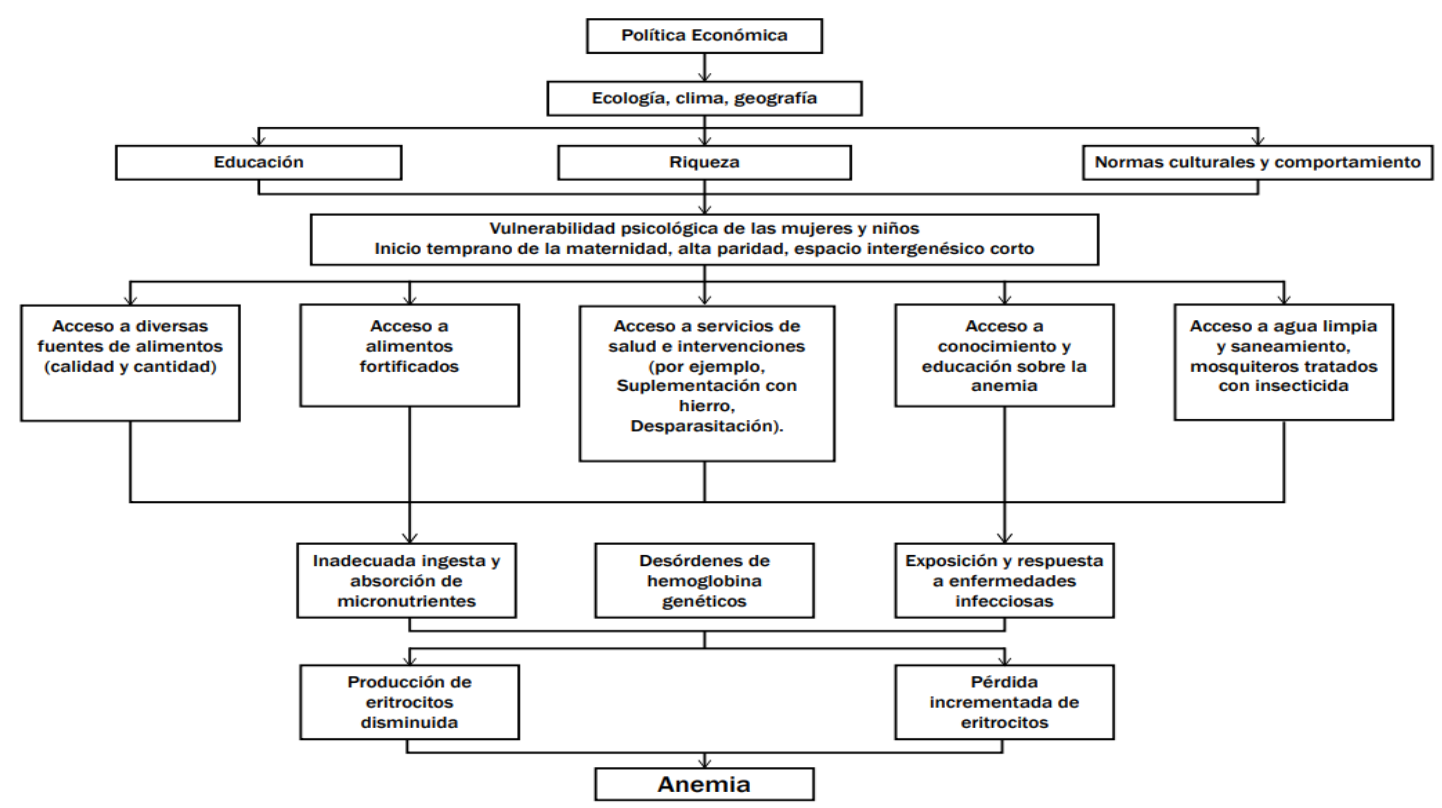

Gráfico 2. Modelo Causal de la Anemia en el Perú

Fuente: Balarajan Yarlini, Lancet 2011

\section{Análisis Normativo}

Es importante considerar que la evolución de las variables de estudio en el caso peruano han tenido un soporte de políticas orientadas a reducir la desnutrición y cuyo efecto o impacto se mide más adelante a través del modelo econométrico propuesto. Sin el ánimo de ser redundante y más bien con el deseo de ilustrar al amable lector a continuación se menciona los principales dispositivos legales en el periodo de estudio: el DS No 001-2001PROMUDEH mediante el cual se conforma la mesa de concertación para la lucha contra la pobreza el cual incluía a 14 millones de peruanos; la Ley N.o 28749/OSINERGMIN que tuvo como objeto hacer que el acceso a la electrificación sea una norma que desarrolle de manera eficiente y sostenible las localidades más alejadas y fronteras del país para elevar y mejorar la calidad de vida, mitigar a la pobreza y fomentar el desarrollo; el DL N.0 1062, Ley de Inocuidad de los Alimentos, el cual tuvo como objetivo asegurar que los alimentos asignados para el consumo humano no tengan efectos perjudiciales para la salud; el DS N.0 003-2008-SA/MINSA, el cual autorizó un listado de intervenciones para garantizar el adecuado servicio sanitario; la Resolución Ministerial N. 0 990-2010-MINSA que aprobaba la Norma Técnica de Salud para el Control del Crecimiento y Desarrollo del Niño menor de Cinco Años; el DS N. ${ }^{\circ}$ 008-2013-MIDIS el cual aprueba la estrategia "Incluir para Crecer"; la Ley de Alimentación Saludable para Niños, Niñas y Adolescentes la cual tuvo como objetivo proteger y promover el derecho que tienen las personas de crecer, desarrollarse y tener acceso a la salud pública en condiciones adecuadas; el Decreto Legislativo N. ${ }^{0} 1164$ el cual amplia la cobertura de salud a través del seguro integral de salud (SIS); la RM N.º 0709-2014-MINAGRI. 


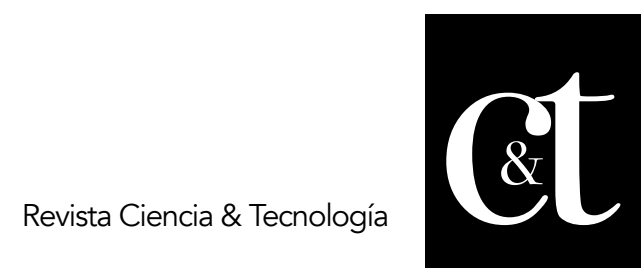

No. 30, 30 de abril de 2021

ISSN impreso: 1390 - 6321

ISSN online: 2661 - 6734

Esta última aprueba los Lineamientos de Política Agraria del Ministerio de Agricultura y Riego para apoyar la seguridad alimentaria ;el DS N. 023-2014 MINAGRI, el cual establece disposiciones que permiten agilizar procesos administrativos para la obtención de licencias de uso del agua en proyectos de inversión, así como darle prioridad de acceso al agua a la población de bajos recursos; la RM N.o 260-2014/MINSA, el cual aprueba a los grupos de apoyo comunal para promover y proteger la lactancia materna; el DL N.0 1240 con el fin de mejorar las normas de los prestadores y usuarios del servicio de saneamiento, así como lineamientos en la instalación de los servicios de saneamiento tanto en zonas rurales como urbanas; se dictó la RM N.o 2912006/MINSA que aprueba la Guía de Práctica Clínica Diarrea Aguda y Cólera en la Niña y el Niño establece estrategias para optimizar la atención sanitaria y reducir los niveles de morbilidad que están asociadas a la diarrea aguda en niños menores de cinco años, mediante un diagnóstico temprano para su tratamiento adecuado ; el artículo 70-A dentro de la Ley N.0 30588-2017, el cual es una reforma constitucional, la cual reconoce al acceso al agua, como un derecho; el DS N.0 068-2018-PCM, el cual declara como prioridad, luchar contra la anemia infantil en niños menores de 36 meses.

En el Perú (véase Gráfico 3), la prevalencia de desnutrición crónica infantil (DCI) en niños entre uno y cinco años disminuyó de $30.1 \%$, en el 2007, a $12.2 \%$ en el 2018 , como también el índice de pobreza y el índice de anemia a 42.4 a $20.5 \%$ y 44.2 a $30.6 \%$ respectivamente.

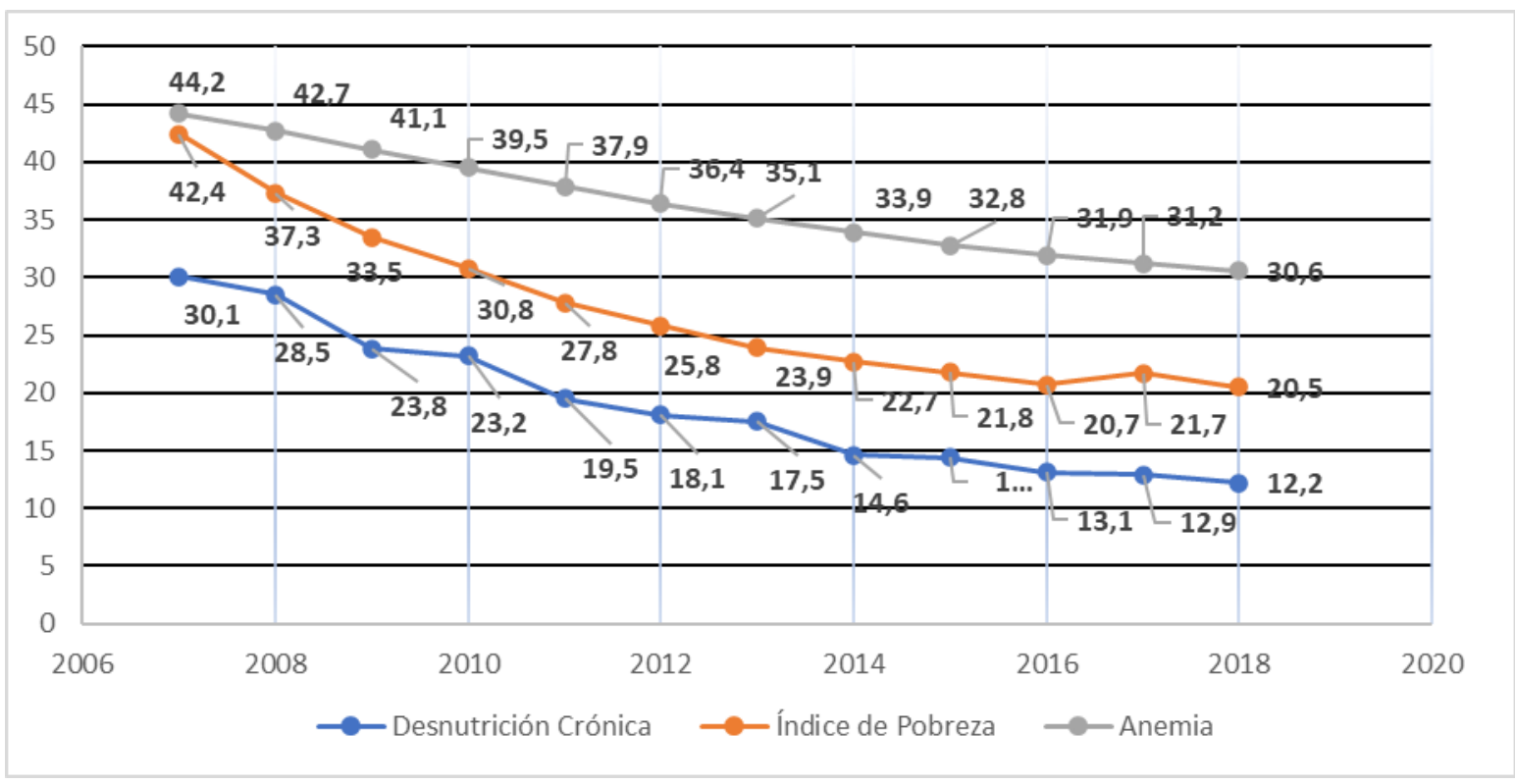

Gráfico 3. Evolución de la desnutrición crónica, pobreza y anemia en el Perú

Fuente: INEI 


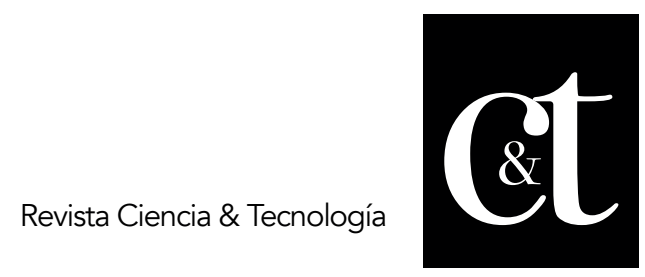

No. 30, 30 de abril de 2021

ISSN impreso: 1390 - 6321

ISSN online: 2661 - 6734

El Ministerio de Salud (2017), señala haber logrado un avance importante en la lucha contra la desnutrición y la anemia infantil. Los cuales se encuentran dentro del plan multisectorial de lucha contra la desnutrición infantil, que actualmente se va desarrollando a nivel nacional a través del cuidado en la higiene y fomentando una adecuada alimentación, especialmente en madres gestantes de primeros infantes.

\section{Desarrollo de la literatura}

Como se puede apreciar hay una efectiva lucha contra la desnutrición en el Perú, sin embargo lo que interesa es presentar un modelo econométrico que permita medir el efecto de las principales variables a partir de un modelo que comprenda variables de : salud, infraestructura y sociales que hayan sido considerados en importantes estudios realizados tanto en el ámbito nacional como internacional .

A continuación dichos estudios son brevemente resumidos en lo concerniente a sus principales objetivos y conclusiones.

Alcázar (2007) señala que el diseño de los programas de lucha contra la desnutrición y anemia estuvo mal diseñado en función de cuál es el objetivo principal ya que hubo una confusión entre aliviar la pobreza, la seguridad alimentaria, su suplementación y el fomento del sector productivo local, cuando el objetivo era reducir la desnutrición. Los programas articulados padecieron al principio de deficiencias en sus componentes dentro del área de educación y salud.

El estudio de Mesinas (2009), referido a los factores socioeconómicos que explican las desigualdades de los niños en el Perú, señala que existe una fuerte relación entre la desnutrición y los grupos de menor poder adquisitivo y precisa que el $63.5 \%$ de los niños desnutridos en el periodo 2004-2007 estaban ubicados en el $38 \%$ de los hogares más pobres del país.

La investigación de Cuycaposa (2010), destaca el hecho de que los niños de la sierra cuyas familias pertenecen al quintil inferior tienen más posibilidades de padecer desnutrición o anemia, que los niños del quintil superior dentro de la misma región. El autor señala en sus conclusiones que el $65 \%$ de los niños pertenecen a familias pobres y el $50 \%$ de ellos tienen un nivel de nutrición por debajo de los niveles normales para su edad.

La investigación de Beltrán y Seinfeld (2011) resalta la importancia de conocer la brecha de ingresos y los quintiles a los cuales pertenece la población. Considera que en los dos quintiles inferiores en el grupo de estudio : el acceso al agua potable, material del piso, el saneamiento, y el material combustible utilizado para la cocina se ubican entre las elementos más significativos para la evaluación de la desnutrición.

El estudio de Alcázar, et al., (2013), tuvo como objetivo estimar el impacto económico de la desnutrición en el Perú para el año 2011, concluye que la anemia tiene un costo para la sociedad peruana aproximadamente S/. 2, 777 millones y que representa aproximadamente el 0,62\% del PBI. 


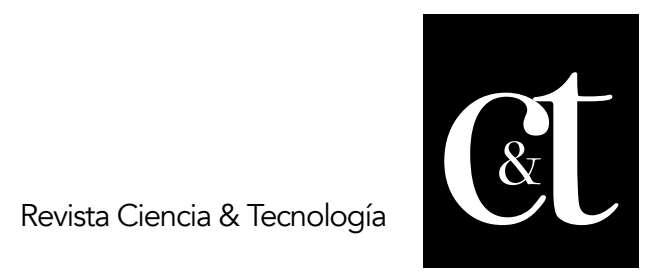

No. 30, 30 de abril de 2021

ISSN impreso: 1390 - 6321

ISSN online: 2661 - 6734

La investigación realizada por Dueñas (2018), evalúa el Impacto del acceso a infraestructura en la pobreza monetaria de los hogares del departamento de Cusco, concluye que las cuatro variables: electricidad, saneamiento, agua potable y telefonía permiten explicar la reducción de la pobreza monetaria y entre ellas el acceso al saneamiento y la telefonía son los que tienen mayor significancia.

El estudio realizado por Fernández, et al., (2017), tuvo como objetivo analizar el estado de las variables seguridad nutricional y alimentaria de Chile, Ecuador y México. Concluyen que la malnutrición se relaciona diferentemente en función de los distintos tipos de desigualdades existentes en América Latina. Por ello las políticas deben ser diferenciadas en cada país considerando el tipo y el nivel de concentración de la desnutrición en las distintas áreas geográficas, y en los grupos vulnerables.

El informe del Ministerio de Salud (2017) menciona que la anemia es un problema que no se limita por estratos socioeconómicos, pero existe una diferencia en promedio de $50 \%$ más, de casos en niños que pertenecen al quintil inferior en relación al quintil superior. Su principal causa: la deficiencia de hierro, además de infecciones crónicas, intoxicación por metales pesados, la parasitosis y las enfermedades hereditarias que afecten la generación de glóbulos rojos.

El estudio realizado por Neyra (2018), analiza el impacto de la inversión pública en infraestructura de saneamiento y agua en el crecimiento económico del Perú , concluye que la inversión pública en infraestructura de agua y saneamiento tiene un efecto positivo en el crecimiento Regional del Perú durante el periodo 20042015. A continuación desarrollamos la literatura de las variables que serán empleadas en el modelo.

\section{Niños con desnutrición crónica menores de cinco años.}

La inseguridad alimentaria en el Perú trae como consecuencia entre otros factores la desnutrición crónica al año 2017 , en la población menor de cinco años de edad pasó de $14,4 \%$ a $13,1 \%$, a nivel nacional, sin embargo afectó principalmente a los departamentos más pobres del país como Huancavelica, Cajamarca, Ucayali, Pasco y Loreto, con tasas de desnutrición crónica superiores al $23,0 \%$.

\section{Prevalencia de la Anemia en niños entre 0 y 5 años de edad}

Investigaciones experimentales y observacionales como la de Zavaleta (2017), exponen a la anemia como principal causante del bajo rendimiento psicomotor del cual forma parte la desnutrición. A pesar que se pueda luchar contra la anemia los efectos en el largo plazo se mantienen y se reflejan en un desempeño inferior en el área social, emocional y cognitiva. La ingesta de hierro en cantidades adecuadas es fundamental para evitar llegar a padecer de anemia. Se debe tener en claro que los niños en crecimiento necesitan mayores proporciones de nutrientes y por ello el seguimiento desde el nacimiento y su desarrollo hasta la adultez es fundamental para mejorar como sociedad. 


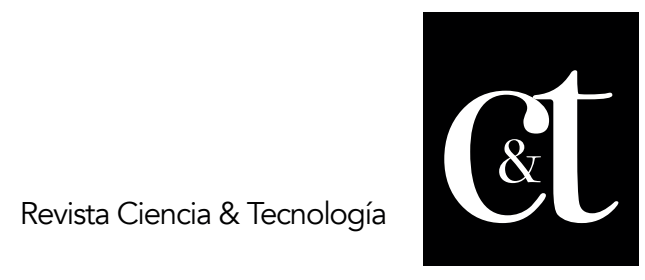

No. 30, 30 de abril de 2021

ISSN impreso: 1390 - 6321

ISSN online: 2661 - 6734

La niñez es la etapa más importante y clave en el desarrollo de una persona, en la que completa su formación neuronal, por lo que el estímulo y el cuidado son esenciales para que alcancen el desarrollo psicomotor y cognitivo necesario que le permita desenvolver su máxima capacidad en la adultez. La anemia infantil, se presenta en mayor grado en niños menores de tres años. Lo cual genera un trastorno que afecta de manera negativa el desarrollo psicomotor y cognitivo de los niños. Crea un obstáculo en sus habilidades para la formación de su personalidad, su integridad y posterior desarrollo de vida digna. Los efectos de padecer anemia se extienden hasta la vida adulta.

\section{Prevalencia de diarrea en niños menores de cinco años}

Según la Dra. Estigarribia (2010) la tercera causa de muerte en el mundo infantil es el círculo que se da entre la diarrea crónica, la mala absorción intestinal de los nutrientes y la desnutrición, esta se constituye en la tercera causa de muerte en el mundo infantil, dicho circulo se perpetua cuando en el entorno social se mantiene la falta de condiciones higiénicas.

\section{Acceso al servicio eléctrico dentro de su vivienda}

La electricidad es una fuerza poderosa, indispensable en nuestra vida diaria. A nivel doméstico es muy útil, e indispensable en el terreno empresarial o en el de la salud. Permite establecer mejores condiciones y cuidados en el control y monitoreo de las enfermedades y cuidados necesarios, tanto para la ingesta de alimentos como para el moderno cuidado progresivo de la salud de la salud. En muchos estudios se ha constituido en un factor importante no solo del atraso económico, sino también de las enfermedades a nivel rural.

\section{Acceso de agua potable dentro de sus viviendas}

Según Rodríguez (2019), el Tribunal Constitucional, a través de la norma dispuesta $N^{\circ}$ 6534-2006/PA/TC, reconoce que todo ciudadano tiene derecho a que el agua potable llegue a sus hogares para su consumo humano. El cuál es un insumo insustituible ya que sólo se puede encontrar en el medio ambiente. Además de ser el recurso más limitado, por las condiciones meteorológicas y los pozos de almacenamiento fluvial, el $40 \%$ de las principales causas de anemia y desnutrición en el Perú se deben al acceso restringido del agua, higiene y saneamiento.

\section{Acceso al servicio de saneamiento dentro de sus hogares}

Chávez (2019), señala que en el Perú, el 35\% de la población carece del servicio de saneamiento. Añade que este servicio es insostenible debido a que los operadores del servicio trabajan con un modelo deficiente de gestión. A ello se suma la falta de apoyo del gobierno central y la innecesaria carga regulatoria. Frente a la problemática el gobierno central se propuso como meta alcanzar la cobertura total del sector urbano para el 2021 y para el año 2030 logar la cobertura en todo el país. Con ello se hizo el intento de alinearse con la política de estado sobre el uso de recursos hídricos y alinearse con los Objetivos de Desarrollo Sostenible (ODS) de la ONU planteados para el año 2030. 


\section{Importancia del quintil inferior}

Según Villanueva (2018), existen factores que determinan los niveles socioeconómicos como el acceso a los servicios básicos, siendo éste el primer paso en la lucha contra la anemia y desnutrición. Es el quintil inferior el más afectado por estas deficiencias gubernamentales y por la baja calidad de los servicios de salud principalmente en las zonas rurales. Pese a ser el grupo que exige una mayor demanda de centros de salud, no fueron adecuadamente atendidos. Según la Organización para las Naciones Unidas para la Alimentación (2018), cuanto mayor sea la disparidad de la distribución de activos como tierras, agua, capital, educación y salud, la barrera para lograr un crecimiento económico para los pobres será más alta y por lo tanto el ritmo de progreso en la reducción de la desnutrición será cada vez más lento.

\section{Importancia de las zonas rurales}

Huerta (2017), explica que los efectos de la desnutrición crónica son graves en el desarrollo de los niños. El retraso en el crecimiento incide más en las áreas rurales que las urbanas. En algunas provincias del Perú, la prevalencia de la desnutrición crónica infantil se da en la zona rural, superando en $20 \%$ a las zonas urbanas.

\section{Metodología}

La investigación fue no experimental de tipo panel con efectos aleatorios, se tomaron en cuenta a todas las regiones del Perú para el periodo 2007 al 2018, las pruebas estadísticas y econométricas utilizas fueron las siguientes: correlación, multicolinealidad, heterocedasticidad, Haussman, $p$ valor, $F, R$ cuadrado, entre otras; se superaron todos los coeficientes que exigen dichas pruebas. Se usaron datos del Ministerio de Salud, Ministerio de Economía y Finanzas y las publicaciones de las encuestas de ENDES a través del portal del Instituto Nacional de Estadística e Informática (INEI).

\section{Modelo}

Se tomaron como estudios referenciales para la selección de variables del modelo (considerando la disponibilidad de información) a los siguientes autores: Alcázar (2007), Mesinas (2009), Cuycaposa (2010), Beltrán y Seinfeld, (2011), Alcázar, et al., (2013), Dueñas (2018), Fernández et al. (2017), Neyra (2018), Zavaleta (2017), y Chávez, R. (2019)

el modelo seleccionado fue:

$$
\begin{aligned}
& \text { DESNUTC } \left.=\beta_{0}+\beta_{1}(\text { ANEM })+\beta_{2} \text { (DIANIN }\right)+\beta_{3}(\text { ELECTR })+\beta_{4}(\text { AGUAD })+ \\
& \beta_{5}(\text { SANITD })+\beta_{6}(\text { QINF })+\beta_{7}(\text { PRUR })
\end{aligned}
$$

En donde:

DESNUTC $=$ Porcentaje de niños con desnutrición crónica menores de cinco años.

ANEM = Prevalencia de anemia en niños menores de cinco años.

DIANIN $=$ Prevalencia de diarrea en niños menores de cinco años

ELECTR $=$ Porcentaje de personas con acceso al servicio eléctrico dentro de su vivienda. 


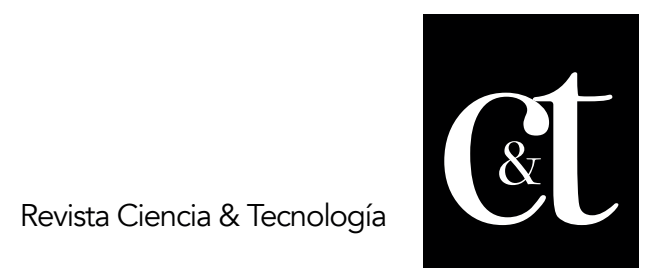

No. 30, 30 de abril de 2021

ISSN impreso: 1390 - 6321

ISSN online: 2661 - 6734

AGUAD = Porcentaje de personas con acceso al agua potable dentro de sus viviendas.

SANITD $=$ Porcentaje de personas con acceso al servicio de saneamiento dentro de sus viviendas

QINF = Porcentaje de personas que se ubican en el quintil inferior.

PRUR $=$ Porcentaje de personas que viven en la zona rural.

\section{Hipótesis}

La hipótesis general de la investigación fue la siguiente: El modelo econométrico panel data elaborado que comprende el periodo 2007 - 2018 es estadísticamente significativo y favorece el análisis de las variables significativas que causan la desnutrición crónica infantil en el Perú.

Las hipótesis específicas fueron:

- La anemia es una causa de la desnutrición crónica en la salud de los niños y su evolución incide desfavorablemente en la reducción de la desnutrición crónica.

- El tratamiento que se ha venido realizando para la diarrea crónica ha sido favorable. Sin embargo, su evolución ha incidido de forma desfavorable para la disminución crónica.

- El incremento en los servicios de electricidad, agua potable y saneamiento ha incidido favorablemente en la reducción de la desnutrición crónica.

- Las políticas de pobreza que han incidido en el quintil inferior han contribuido desfavorablemente en la reducción de la desnutrición crónica.

- El incremento del nivel de personas que viven en las zonas rurales ha incidido desfavorablemente en la reducción de la desnutrición crónica.

La investigación es no experimental de tipo panel se tomaron en cuenta a todas las regiones del Perú para el periodo 2007 al 2018. Se usaron datos del Ministerio de Salud, Ministerio de Economía y Finanzas y las publicaciones de las encuestas de ENDES a través del portal del Instituto Nacional de Estadística e Informática (INEI).

\section{Resultados y discusión}

Por los resultados econométricos obtenidos se ha logrado probar todas las hipótesis planteadas, en primer lugar se puede precisar que el modelo econométrico panel es estadísticamente significativo los valores de los parámetros $\mathrm{p}$ - valor así lo demuestran. Asimismo cada hipótesis especifica planteada ha sido probada como explicamos a continuación a través de las siguientes variables, véase la tabla $\mathrm{N}^{\circ} 1$ : 


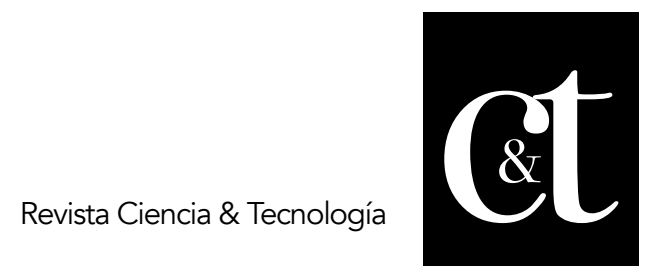

No. 30, 30 de abril de 2021

ISSN impreso: 1390 - 6321

ISSN online: 2661 - 6734

Tabla 1. Resultados de las pruebas econométricas, efectos aleatorios

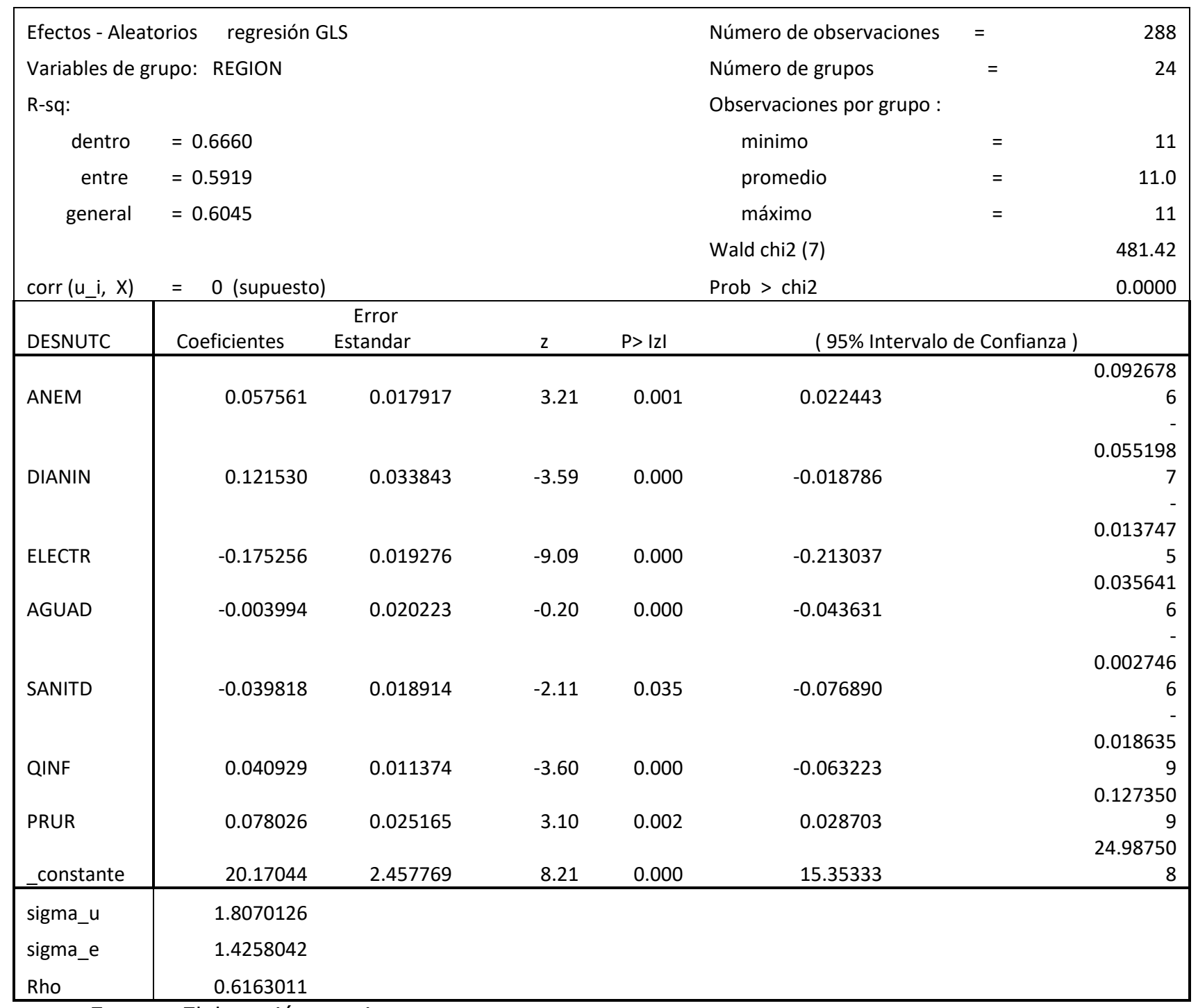

Fuente: Elaboración propia

La prevalencia de anemia en niños menores de cinco años (ANEM), resultó significativa y con signo positivo lo que significa que por cada punto porcentual adicional de anemia se incrementa la variable DESNUTC en 0.05761 puntos porcentuales.

El nivel de diarrea crónica en niños menores de cinco años (DIANIN), resultó significativo y con signo positivo lo que significa que por cada punto porcentual adicional de dicha variable se incrementa la variable DESNUTC en 0.121530 . Obsérvese que la diarrea crónica tiene en el modelo mayor incidencia que la anemia. 


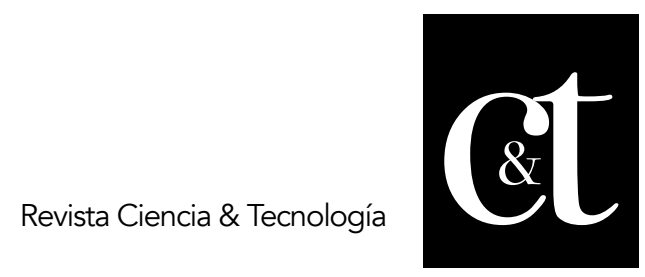

No. 30, 30 de abril de 2021

ISSN impreso: 1390 - 6321

ISSN online: 2661 - 6734

Personas que cuentan con electrificación en el hogar (ELECTR), resultó significativa y con signo negativo frente a la desnutrición crónica, debido a que al contar con mayor electricidad las madres y la familia pueden hacer uso de más horas en sus actividades cotidianas de aseo y cuidado a los niños. El acceso al servicio de electricidad reduce el nivel de desnutrición por ser un determinante importante en el nivel de educación, nivel de pobreza y calidad alimentaria. Por cada punto porcentual adicional de personas con acceso al servicio de electricidad dentro de su hogar se reduce la variable DESNUTC en - 0.175256 puntos porcentuales.

Personas con agua potable dentro del hogar (AGUAD) ,resultó ser significativa con signo negativo respecto a la desnutrición crónica en niños, al contar con mayor agua potable dentro del hogar se incrementa los niveles de aseo y a través de ella se reduce los niveles de desnutrición crónica infantil. El uso de aguas fluviales puede ocasionar enfermedades infecciosas, ya que en muchas regiones del Perú los ríos están contaminados. Por cada punto porcentual adicional de personas con acceso al agua potable dentro de sus viviendas se reduce la variable DESNUTC en -0.003994 puntos porcentuales.

Personas con el servicio de saneamiento dentro de sus hogares (SANITD) es significativa con signo negativo lo cual es congruente respecto a que a mayor saneamiento menor nivel de desnutrición crónica. El mayor acceso a saneamiento disminuye los niveles de desnutrición ya que mitigan el efecto de contaminación por residuos orgánicos y falta de limpieza. Por cada punto porcentual adicional de personas con acceso al servicio de saneamiento dentro de sus viviendas se reduce la variable DESNUTC en -0.039818 puntos porcentuales. Obsérvese que de los 3 servicios públicos analizados, en el modelo evaluado el servicio de electrificación tiene un mayor coeficiente que los servicios de saneamiento y agua.

Personas que pertenecen al quintil inferior (QINF), es significativa con signo positivo lo cual sugiere que mientras más personas se encuentren en este quintil, mayor será el nivel de desnutrición crónica. Este segmento de la población tiene restricciones alimentarias por dificultades de acceso a mercados y a los altos costos de transportes de mercaderías y alimentos. El efecto sobre la variable DESNUTC es 0.040929 puntos porcentuales.

Personas que viven en las zonas rurales (PRUR), es significativa con signo positivo en relación al nivel de desnutrición crónica. Este resultado se debe a las carencias en las que viven las familias en las zonas rurales donde existen barreras para el acceso a centros de salud, agua, luz y educación. Por cada punto porcentual adicional en la variable personas que pertenezcan a la población rural ,se incrementa la variable DESNUTC en 0.078026 puntos porcentuales. Obsérvese finalmente que las variables sociales : quintil inferior y zonas rurales afectan positivamente a la variable desnutrición corroborando la literatura que se desarrollo tanto por organismos internacionales como por diferentes autores.

Las opiniones y recomendaciones de expertos en el tema de la desnutrición infantil y las demás variables seleccionadas que explican su impacto en el tiempo han sido claves en el desarrollo de esta investigación.

Villamonte, Huaman. Evaluación de los determinantes de la desnutrición crónica en el Perú 


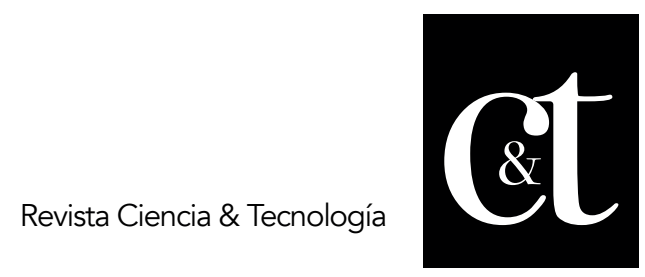

No. 30, 30 de abril de 2021

ISSN impreso: 1390 - 6321

ISSN online: 2661 - 6734

Se ha comprobado a través del presente estudio : la relación de causalidad que tiene la desnutrición infantil con el nivel de pobreza (UNICEF,1998) y, la importancia de las variables de estudio seleccionadas que consideraron también en sus estudios, Garcia L. (2002), Mesinas (2009), Cuycaposa (2010), Fernández y Martínez ( 2017) Neyra (2018).

\section{Conclusiones}

El propósito de la investigación fue analizar la significancia de las variables determinantes de la desnutrición crónica infantil propuestas por la UNICEF (1998), para el caso de Perú elaborando un modelo panel data en base a datos publicados por el INEI.

Se ha logrado probar todas las hipótesis planteadas, se puede precisar que el modelo econométrico panel es estadísticamente significativo .

Todas las variables empleadas son significativas una breve comparación entre ellas permite apreciar que la diarrea crónica tiene en el modelo mayor incidencia que la anemia en la reducción de la desnutrición, como también que de los 3 servicios públicos de infraestructura analizados, el servicio de electrificación tiene un mayor coeficiente de incidencia que los servicios de saneamiento y agua, finalmente el quintil inferior y las zonas rurales afectan positivamente a la variable desnutrición corroborando la literatura empleada.

Se puede apreciar que en el caso peruano las diferentes políticas del sector salud como de los sectores vinculados al desarrollo de la salud viene logrando una efectiva lucha contra la reducción de la desnutrición

Se recomienda que las autoridades públicas consideren la significancia del modelo y que le sirva de referencia para el perfeccionamiento de los dispositivos legales de política de salud vigentes y de las tareas de control y monitoreo necesarias.

Que todos los sectores involucrados articulen con mayor exigencia sus proyectos y acciones de intervención brindando preferencia a la zonas rurales y al quintil inferior lo cual conducirá a una reducción franca y sostenible lucha contra la desnutrición.

Se recomienda que las universidades fomenten el desarrollo de estudios vinculados al mejoramiento de la salud principalmente con la participación de todas las facultades por cuanto hoy en día el trabajo multidisciplinario brinda nuevos enfoques y soluciones sostenibles.

Se recomienda no claudicar en el reconocimiento de la función educativa permanente de carácter preventivo, en la cual deben participar: funcionarios públicos, médicos, personal de salud, educadores, padres e hijos.

\section{Referencias}

Alcázar, L. (2012). Impacto económico de la anemia en el Perú. (A. c. hambre, Ed.) Obtenido http://www.grade.org.pe/upload/publicaciones/archivo/download/pubs/LIBR OGRADE_ANEMIA.pdf

Alcázar, L. (2007). ¿Por qué no funcionan los programas alimentarios y nutricionales en el Perú? Riesgos y oportunidades para su reforma. Lima: Grade. 


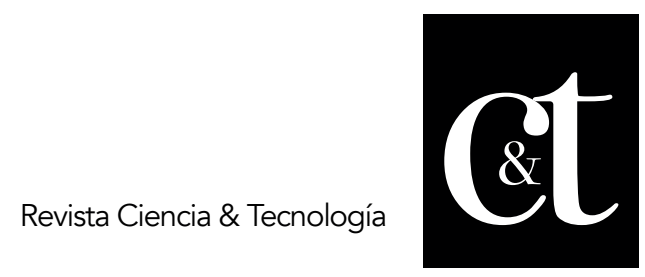

No. 30, 30 de abril de 2021

ISSN impreso: 1390 - 6321

ISSN online: 2661 - 6734

Banco Mundial. ( 2018). Combate a la desnutrición en Perú: mejoras en la demanda, oferta y administración de servicios de salud y nutrición en tres regiones. Obtenido de https://www.bancomundial.org/es/results/2018/04/18/fighting-malnutritionin-peru

Beltrán, A. y Seinfeld, J. (2011). Identificando estrategias efectivas para combatir la desnutrición infantil en el Perú. . Obtenido de http://hdl.handle.net/11354/507

Chávez, R. (2019). Agua y saneamiento: Radriogafía de un sector prioritario en el Perú. Obtenido de https://stakeholders.com.pe/informes/aguasaneamiento-radiografia-sector-prioritario-peru/

Cuycaposa, N. (2010). Análisis de equidad del nivel socioeconómico y la desnutrición crónica en niños menores de 5 años en el Perú. Lima: Universidad Nacional Mayor de San Marcos.

Dispositivos Legales: Decretos Legislativos N. ${ }^{\circ} 1062 ; N^{\circ} 30021 ; N^{\circ} 1164 ; N^{\circ}$ 1240 que modifica la ley $\mathrm{N}^{\circ}$ 26338, Ley General de Servicios de Saneamiento y La Ley $\mathrm{N}^{\circ}$ 30045; Decretos Supremos N. ${ }^{\circ}$ 007-2015-SA. ; N. ${ }^{\circ}$ 008-2013-MIDIS; $N^{\circ}$ 001-2001-PROMUDEH; $\mathrm{N}^{\circ}$ 003-2008-SA/MINSA; $\mathrm{N}^{\circ}$ 008-2013-MIDIS; $N^{\circ}$ 012-2018. N 023-2014 MINAGRI ; $N^{\circ}$ 068-2018-PCM. N021; DS N 010-2016-MIDIS; Resoluciónes Ministeriales N. 0709-2014MINAGRI; No 260-2014/MINSA; No 291-2006/MINSA; No 990-2010-MINS

Dueñas Taype, F. (2018). Impacto del acceso a infraestructura en la pobreza monetaria de los hogares del departamento de cusco, 2016. Tesis para optar por el título de Economista. Cusco, Perú. Obtenido de http://repositorio.uandina.edu.pe/handle/UAC/1976

Estigarribia, M. (2010) , Diarrea y desnutrición: una preocupante combinación Obtenido en https://www.ellitoral.com/index.php/diarios/2010/08/31/salud/SALUD01.html

FAO. (2019). El estado de la seguridad alimentaria y la nutrición en el Mundo 2019. Obtenido de http://www.fao.org/3/ca5162es/ca5162es.pdf

Fernández, A., Martínez, R., \& A., C. I. (2017). Impacto Social y económico de la malnutrición. Modelo de análisis y estudio piloto en Chile, el Ecuador y México. CEPAL. Obtenido de http://www.codajic.org/sites/www.codajic.org/files/Impacto\%20social\%20y \%20econ\%C3\%B3mico\%20de\%20la\%20malnutrici\%C3\%B3n\%20.pdf

Huamán Huarcaya, M.A. (2020). Determinantes de la desnutrición crónica en niños de 0 a 5 años en el Perú para el periodo 2007 -2018, Tesis para la obtención del titulo de economista, Universidad de Lima, Lima Perú.

Huerta, E. (2017). La desnutrición crónica afecta al 28\% de niños menores de 5 años de las zonas rurales del Perú. Lima, Perú: Radio Programas del Perú.

Instituto Nacional de Estadísitca e Informática. (2017). Indicadores de resultados de los programas presupuestales 2012-2017. Lima, Perú.: Encuesta Demográfica y de Salud Familiar 2017.

Instituto Nacional de Estadística e Informática. (2018). Resultaos de la pobreza monetaria $2018 . \quad$ Obtenido de https://www.inei.gob.pe/media/cifras de pobreza/exposicion evolucion-depobreza-monetaria-2018.pdf

Villamonte, Huaman. Evaluación de los determinantes de la desnutrición crónica en el Perú 


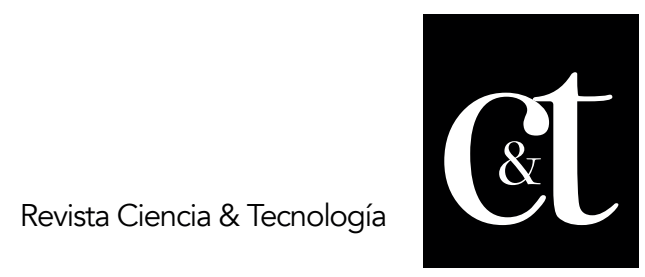

No. 30, 30 de abril de 2021

ISSN impreso: 1390 - 6321

ISSN online: 2661 - 6734

Mesinas, J. M. (2009). Factores socioeconómicos que explican las desigualdades nutricionales de nuestros niños. ¿Por dónde hay que atacar?. . Obtenido de https://determinantes.dge.gob.pe/archivos/FX_socioeconomicos.pdf

Ministerio de Salud. (2017). Plan Nacional para la Reducción de la anemia $2017-$ 2021. Lima, Lima, Perú.

Neyra Lujano, E. (2018). La inversión pública en infraestructura de agua y saneamiento y su efecto en el crecimiento económico del Perú, periodo 2004 - 2015. Tesis para optar por el título profesional de Ingeniero Economista. Universidad Nacional del Altiplano. Puno, Perú. . Obtenido de http://repositorio.unap.edu.pe/handle/UNAP/7565

Organización Mundial de al Salud. (2016). Obtenido de www.who.int/es/newsroom/fact-sheets/detail/malnutrition

Organizacion Mundial de la Salud. (2014). Obtenido de https://www.who.int/nutrition/about_us/es/

Organización Mundial de la Salud. (2017). Metas mundiales de nutrición 2025: Documento normativo sobre anemia. Ginebra. Obtenido de http://www.who.int/nutrition/publications/globaltargets2025_policybrief_ana emia/es/

Organización Panamericana de la Salud. (2003). Salud, crecimiento económico y reducción de la pobreza. Informe del Grupo de Trabajo 1 de la Comisión sobre Macroeconomía y Salud. Pan American Health Org.

Organizacion Panmericana de Salud. (2018). Obtenido de https://www.paho.org/per/index.php?option=com_joomlabook\&view=topic\&i $d=233$

Rodríguez, R. (2019). El derecho al agua potable y la anemia en el Perú. Lima, Perú.

UNICEF. (1998). Estado Mundial de la Infancia 1998. Obtenido de https://www.unicef.org/spanish/sowc98sp/spsw98a.pdf

UNICEF. (2008). Lineamientos Estratégicos para la Erradicación de la Desnutrición Crónica Infantil en América Latina y el Caribe. Panamá: UNICEF TACRO.

Villanueva, C. (2018). Entrevista realizada por Inversión en la Infancia. Lima, Perú: Inversión en la Infancia.

Zavaleta, N. (2017). Anemia infantil: retos y oportunidades al 2021. Lima, Perú.: Revista Peruana de medicina Experimental y Salud Pública. 